\title{
Regression and stabilization of advanced murine atherosclerotic lesions: a comparison of LDL lowering and HDL raising gene transfer strategies
}

\author{
Eline Van Craeyveld • Stephanie C. Gordts • \\ Elena Nefyodova $\cdot$ Frank Jacobs $\cdot$ Bart De Geest
}

Received: 2 October 2010 /Revised: 4 December 2010 / Accepted: 30 December 2010 /Published online: 21 January 2011

(C) The Author(s) 2011. This article is published with open access at SpringerLink.com

\begin{abstract}
Both reductions in atherogenic lipoproteins and increases in high-density lipoprotein (HDL) levels may affect atherosclerosis regression. Here, the relative potential of low-density lipoprotein (LDL) lowering and HDL raising gene transfer strategies to induce regression of complex murine atherosclerotic lesions was directly compared. Male C57BL/6 LDL receptor (LDLr) ${ }^{-/-}$mice were fed an atherogenic diet $(1.25 \%$ cholesterol and $10 \%$ coconut oil) to induce advanced atherosclerotic lesions. A baseline group was killed after 6 months and remaining mice were randomized into a control progression (Adnull or saline), an apolipoprotein (apo) A-I (AdA-I), an LDLr (AdLDLr), or a combined apo A-I/LDLr (AdA-I/AdLDLr) adenoviral gene transfer group and followed-up for another 12 weeks with continuation of the atherogenic diet. Gene transfer with AdLDLr decreased non-HDL cholesterol levels persistently by $95 \%(p<0.001)$ compared with baseline. This drastic reduction of non-HDL cholesterol levels induced lesion regression by $28 \%(p<0.001)$ in the aortic root and by $25 \%(p<0.05)$ in the brachiocephalic artery at 12 weeks after transfer. Change in lesion size was accompanied by enhanced plaque stability, as evidenced by increased collagen content, reduced lesional macrophage content, a drastic reduction of necrotic core area, and decreased expression of inflammatory genes. Elevated HDL cholesterol following AdA-I transfer increased collagen content in
\end{abstract}

Electronic supplementary material The online version of this article (doi:10.1007/s00109-011-0722-x) contains supplementary material, which is available to authorized users.

E. Van Craeyveld · S. C. Gordts · E. Nefyodova · F. Jacobs ·

B. De Geest $(\triangle)$

Center for Molecular and Vascular Biology, University of Leuven,

Campus Gasthuisberg, Herestraat 49,

3000 Leuven, Belgium

e-mail: bart.degeest@med.kuleuven.be lesions, but did not induce regression. Apo A-I gene transfer on top of AdLDLr transfer resulted in additive effects, particularly on inflammatory gene expression. In conclusion, drastic lipid lowering induced by a powerful gene transfer strategy leads to pronounced regression and stabilization of advanced murine atherosclerosis.

Keywords Atherosclerosis - Advanced lesions .

Regression · Plaque stabilization - Adenoviral gene transfer. Lipoproteins

\section{Introduction}

The primary target for prevention of cardiovascular morbidity and mortality is dyslipidemia [1]. Lipid lowering attenuates inflammatory stimuli within complex atherosclerotic lesions leading to plaque stabilization [2]. In addition, very intensive lipid lowering with rosuvastatin has been associated with regression of atherosclerosis in the ASTEROID trial [3]. Whereas the change in low-density lipoprotein (LDL) cholesterol is predictive of the change in atheroma volume, it has been postulated that statins may affect lesion regression via pleiotropic, lipid loweringindependent effects $[4,5]$. Therefore, it remains to be demonstrated that aggressive lipid lowering itself is sufficient to induce regression of atherosclerosis.

Plasma levels of high-density lipoprotein (HDL) cholesterol and its major apolipoprotein (apo), apo A-I, are inversely correlated with the risk of ischemic cardiovascular diseases [6]. Apart from their lipid lowering properties, statins have been reported to increase HDL cholesterol levels by $5 \%$ to $15 \%[3,7]$. A recent meta-analysis of IVUS trial data [7] indicated that statin therapy is associated with regression of coronary atherosclerosis when LDL choles- 
terol is substantially reduced and HDL cholesterol is increased by more than $7.5 \%$. The addition of extendedrelease niacin to statin therapy significantly increased HDL cholesterol and slowed the progression of carotid atherosclerosis among individuals with known coronary heart disease [8]. Furthermore, pilot intervention studies in humans using infusions of both apo A- $\mathrm{I}_{\text {Milano }}$ [9] and wild-type human apo A-I [10] containing phospholipid complexes show promising but not unequivocal results on imaging end-points. These observations raise the question whether a combination of an LDL lowering and HDL raising approach could be the most effective intervention for establishing regression of atherosclerosis.

There is a paucity of experimental animal models that evaluate regression of advanced atherosclerotic lesions. Indeed, although regression of fatty streaks and intermediate lesions has been convincingly demonstrated in different animal models $[11,12]$, these lesions are not very relevant from a clinical point of view. Furthermore, lipid lowering in prior experimental regression studies of advanced atherosclerosis included drastic manipulations of the experimental diet $[4,13-15]$, which may result in effects that occur independent of cholesterol lowering.

Based on a series of studies directed at a continuous improvement of expression cassettes for hepatocytedirected gene transfer [16-18], we developed an E1E3E4deleted adenoviral LDL receptor (LDLr) expressing vector that induces a more than $95 \%$ persistent reduction of nonHDL cholesterol in hyperlipidemic mice notwithstanding continuation of the atherogenic diet. Our first objective was to investigate the degree of regression of advanced lesions with clearly developed necrotic cores induced by this LDLr expressing vector. Secondly, to further elucidate the relative contributions of reductions in atherogenic lipoproteins and increases in HDL cholesterol on the progression/regression rate of advanced atherosclerotic lesions, we compared atherosclerosis in mice treated with LDLr, with apo A-I, or with combined apo A-I/LDLr gene transfer. Our results show that the drastic reduction of non-HDL cholesterol levels after AdLDLr gene transfer induced stabilization and powerful regression of advanced, preexisting lesions. Increased HDL cholesterol following apo A-I gene transfer augmented collagen content in lesions, but did not induce regression. Gene expression profiling indicated that apo A-I gene transfer on top of AdLDLr transfer resulted in additive effects, particularly on inflammatory gene expression.

\section{Materials and methods}

Generation of recombinant adenoviral vectors The E1E3E4-deleted adenoviral vector AdLDLr contains the
$1.2 \mathrm{~kb}$ DC172 promoter [18], consisting of an $890 \mathrm{bp}$ human $\alpha_{1}$-antitrypsin promoter and two copies of the $160 \mathrm{bp} \alpha_{1}$-microglobulin enhancer, upstream of the $5^{\prime}$ untranslated region (UTR) of the human apo A-I gene that contains the first intron, and upstream of the $2.6 \mathrm{~kb} L D L r$ sequence [19] and two copies of the 774 bp hepatic control region-1. Following an EcoRI restriction, the 5' UTR of the human apo A-I gene together with the $2.6 \mathrm{~kb} L D L r$ cDNA sequence was isolated from pGEM-A-Iintron-LDLr [19] and subsequently ligated into the EcoRI site of pTG15203$\mathrm{DC}_{172 . H C R}$ [18]. Construction of the E1E3E4-deleted adenoviral vector AdA-I containing the DC172 promoter upstream of the genomic human apo $A-I$ sequence and two copies of HCR-1 has been described before [18]. The E1E3E4-deleted control vector Adnull does not contain an expression cassette. Construction of infectious plasmids and large-scale production of recombinant E1E3E4-deleted adenoviral vectors was performed as described previously [20].

Animal studies- All experimental procedures were approved by the Institutional Animal Care and Research Advisory Committee of the University of Leuven. C57BL/6 $\mathrm{LDLr}^{-/}$ mice were purchased from Jackson Laboratories (Bar Harbor, ME, USA). At the age of 3 months, male C57BL/ $6 \mathrm{LDLr}^{-/-}$mice received an atherogenic diet containing $1.25 \%(w / w)$ cholesterol and $10 \%(v / w)$ coconut oil ad libitum. This diet was maintained throughout the entire experiment. Six months after start of the diet, a baseline reference group was euthanised for histological analysis. Control progression mice were injected via the tail vein with $1 \times 10^{11}$ adenoviral particles of the control vector Adnull, which does not contain an expression cassette, or alternatively with saline buffer. Intervention groups were treated with $5 \times 10^{10}$ adenoviral particles of AdA-I (AdA-I group), $5 \times 10^{10}$ adenoviral particles of AdLDLr (AdLDLr group), or $5 \times 10^{10}$ adenoviral particles of AdA-I combined with $5 \times 10^{10}$ adenoviral particles of AdLDLr (AdA-I/AdLDLr group). Mice in the AdA-I and AdLDLr groups were co-injected with $5 \times 10^{10}$ particles of Adnull to avoid differences in the total vector dose. Blood was collected after an overnight fast by puncture of the retro-orbital plexus and plasma was immediately isolated by centrifugation at $1,100 \mathrm{~g}$ for $10 \mathrm{~min}$ and stored at $-20{ }^{\circ} \mathrm{C}$. Twelve weeks after gene transfer or saline injection, mice of the control progression group or interventions groups were killed for histological analysis.

Plasma lipid analysis Please see "Materials and methods" in the Electronic supplementary materials.

Human apo A-I enzyme immunoassay Please see "Materials and methods" in the Electronic supplementary materials. 
Histological analysis Please see "Materials and methods" in the Electronic supplementary materials.

Gene expression profiling Please see "Materials and methods" in the Electronic supplementary materials.

Statistical analysis All data are expressed as means \pm standard error of the mean (SEM). Areas under the curve were calculated using Prism4 (GraphPad Software, San Diego, CA, USA). Lipid and histological parameters were compared by analysis of variance followed by Tukey's multiple comparison post-test or when indicated by Kruskal-Wallis test followed by Dunn's multiple comparison post-test using Instat3 (GraphPad Software). A twosided $p$ value of less than 0.05 was considered statistically significant.

\section{Results}

AdLDLr gene transfer significantly lowers non-HDL cholesterol levels whereas AdA-I gene transfer increases HDL cholesterol levels in $\mathrm{LDLr}^{-1-}$ mice

An atherogenic diet containing $1.25 \%(w / w)$ cholesterol and $10 \%(v / w)$ coconut oil was initiated in male C57BL/6
$\mathrm{LDLr}^{-/-}$mice at the age of 3 months. Six months after start of the diet, mice were treated with the human apo A-I expressing vector AdA-I $(n=13)$, the low-density lipoprotein receptor expressing vector $\operatorname{AdLDLr}(n=12)$, or a combination of both vectors (AdA-I/AdLDLr) $(n=12)$. Control progression mice were injected with the control vector Adnull $(n=7)$ or with saline $(n=6)$. Since no difference occurred between the Adnull and saline injected mice, data of both control progression groups were pooled. The atherogenic diet was maintained throughout the entire experiment. Figure $1 \mathrm{a}-\mathrm{c}$ show the time course of total, non-HDL, and HDL plasma cholesterol levels, respectively, in male $\mathrm{C} 57 \mathrm{BL} / 6 \mathrm{LDLr}^{-/-}$mice of the control progression, AdA-I, AdLDLr, and AdA-I/AdLDLr intervention group. Average plasma total cholesterol, non-HDL cholesterol, and HDL cholesterol levels for the 12 week period after gene transfer are shown in Table 1. Gene transfer with AdLDLr and AdA-I/AdLDLr decreased non-HDL cholesterol levels persistently by $95 \%(p<0.001)$ and by $91 \%(p<0.001)$, respectively, compared with baseline (Fig. 1a, Table 1). This major reduction of non-HDL cholesterol occurred in the VLDL, IDL, and LDL fractions, as indicated in Table 1. AdA-I gene transfer resulted in a 1.5 -fold $(p<0.001)$ increase of HDL cholesterol levels without affecting non-HDL cholesterol levels (Fig. 1b-c, Table 1). HDL cholesterol levels in the AdA-I/AdLDLr treatment group were 1.8-fold
Fig. 1 Time course of total plasma cholesterol levels (a), non-HDL plasma cholesterol levels (b), HDL plasma cholesterol levels (c), and total plasma triglyceride levels (d) in male C57BL/6 $\mathrm{LDLr}^{-/-}$mice of the control progression (empty squares) $(n=13)$, and the AdA-I (filled triangles) $(n=13)$, AdLDLr (filled circles) $(n=12)$, and AdA-I/AdLDLr (filled circles) $(n=12)$ intervention groups. An atherogenic diet $(1.25 \%$ cholesterol $(w / w), 10 \%$ coconut oil $(v / w))$ was initiated 26 weeks before adenoviral gene transfer or saline injection and maintained throughout the experiment. The 0 week time point corresponds to the time point of gene transfer in the intervention groups. All data represent means \pm SEM

Table 1 Average total, non-HDL, VLDL, IDL, LDL, and HDL cholesterol levels (mg/dl) and average total triglyceride levels (mg/dl) in plasma of male C57BL/6 LDLr ${ }^{-/}$mice for the 12-week time period in the control progression group, and in AdA-I, AdLDLr, and AdA-I/AdLDLr groups

\begin{tabular}{|c|c|c|c|c|}
\hline & Control progression & AdA-I & AdLDLr & AdA-I/AdLDLr \\
\hline Total cholesterol & $710 \pm 18$ & $750 \pm 13$ & $80 \pm 7.8^{\S \S \S, \# \# \#}$ &  \\
\hline Non-HDL cholesterol & $640 \pm 20$ & $650 \pm 16$ & $30 \pm 3.5^{\S \S \S, \# \# ~}$ & 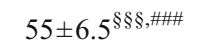 \\
\hline VLDL cholesterol & $150 \pm 14$ & $160 \pm 13$ & 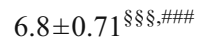 &  \\
\hline IDL cholesterol & $300 \pm 12$ & $300 \pm 8.2$ & 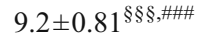 & $16 \pm 1.6^{\S \S \S, \# \# \#}$ \\
\hline LDL cholesterol & $180 \pm 14$ & $180 \pm 8.9$ &  & 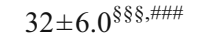 \\
\hline HDL cholesterol & $72 \pm 3.5$ & $108 \pm 6.6^{\S \S \S}$ & 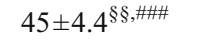 &  \\
\hline Total triglycerides & $290 \pm 47$ & $420 \pm 41^{\S}$ &  &  \\
\hline
\end{tabular}

Data are expressed as means \pm SEM ( $n=13$ for control progression, $n=13$ for AdA-I, $n=12$ for AdLDLr, and $n=12$ for AdA-I/AdLDLr). Lipoproteins were isolated by ultracentrifugation at the time points shown in Fig. 1 and average values for the time period between 1 and

12 weeks after gene transfer were obtained by dividing the area under the curve by the time of follow-up

${ }^{\S} p<0.05 ;{ }^{\S} p<0.01 ;{ }^{\S \S} p<0.001$, versus control progression; ${ }^{\# \#} p<0.01 ;{ }^{\# \#} p<0.001$, versus AdA-I; ${ }^{\text {बा }} p<0.01 ;{ }^{9 \uparrow \uparrow} p<0.001$, versus AdLDLr

$(p<0.001)$ higher compared with levels in AdLDLr treated mice, but not significantly different compared with control progression mice. HDL cholesterol levels in AdLDLr mice were reduced by $37 \%(p<0.01)$ compared with control progression mice (Fig. 1c, Table 1). Human apo A-I levels were $160 \pm 19 \mathrm{mg} / \mathrm{dl}$ and $130 \pm 12 \mathrm{mg} / \mathrm{dl}$ following AdA-I and AdA-I/AdLDLr gene transfer, respectively.

Time course of total plasma triglyceride levels for the four intervention groups is shown in Fig. 1d. Compared with control progression mice, average plasma triglycerides increased 1.4-fold $(p<0.05)$ after AdA-I gene transfer and were reduced by $82 \%(p<0.001)$ and $77 \%(p<0.001)$ after AdLDLr and AdA-I/AdLDLr gene transfer, respectively.



Fig. 2 Average intimal area $\left(\mu \mathrm{m}^{2}\right)$ determined at the aortic root (a) or brachiocephalic artery (b) of male $\mathrm{C} 57 \mathrm{BL} / 6 \mathrm{LDLr}^{-/-}$mice of the baseline group $(n=13)$, killed 26 weeks after feeding the atherogenic diet, of the control progression group $(n=13)$, or of the AdA-I $(n=13)$, the $\operatorname{AdLDLr}(n=12)$, and AdA-I/AdLDLr $(n=12)$ intervention groups
AdLDLr and AdA-I/AdLDLr gene transfer induce pronounced regression of advanced atherosclerotic lesions in the aortic root and brachiocephalic artery of $\mathrm{LDLr}^{-/-}$mice

Figure $2 \mathrm{a}$ and $\mathrm{b}$ show the intimal area at the aortic root and $\mathrm{BCA}$, respectively, in male $\mathrm{C} 57 \mathrm{BL} / 6 \mathrm{LDLr}^{-1-}$ mice of the baseline group $(n=13)$, killed 6 months after feeding the atherogenic diet, and in male $\mathrm{C} 57 \mathrm{BL} / 6 \mathrm{LDLr}^{-/}$mice 12 weeks after transfer with AdA-I $(n=13)$, AdLDLr $(n=12)$, or AdA-I/AdLDLr $(n=12)$, or in the control progression group $(n=13)$. Representative histological sections of atherosclerotic lesions are shown in Fig. 3. Feeding an atherogenic diet to male $\mathrm{C} 57 \mathrm{BL} / 6 \mathrm{LDLr}^{-/-}$mice for a period of 6 months resulted in the development of



12 weeks after gene transfer with continuation of the atherogenic diet. Quantifications were performed on hematoxylin and eosin stained sections at $70 \mu \mathrm{m}$-spaced intervals along the entire aortic root or BCA. Data are expressed as means \pm SEM 
Fig. 3 Representative hematoxylin and eosin stained sections of the aortic root (left: a, c, e, g, and i) and brachiocephalic artery (right: $\mathbf{b}, \mathbf{d}, \mathbf{f}, \mathbf{h}$, and $\mathbf{j}$ ) of male $\mathrm{C} 57 \mathrm{BL} / 6$ $\mathrm{LDLr}^{-/-}$mice of the baseline group (a-b), or of male C57BL/6 $\mathrm{LDLr}^{-/-}$mice of the control progression (c-d), or of the dA-I, (e-f), AdLDLr (g-h), and AdA-I/ AdLDLr (i-j) intervention groups. Scale bar represents $500 \mu \mathrm{m}$ (left) and $200 \mu \mathrm{m}$ (right)
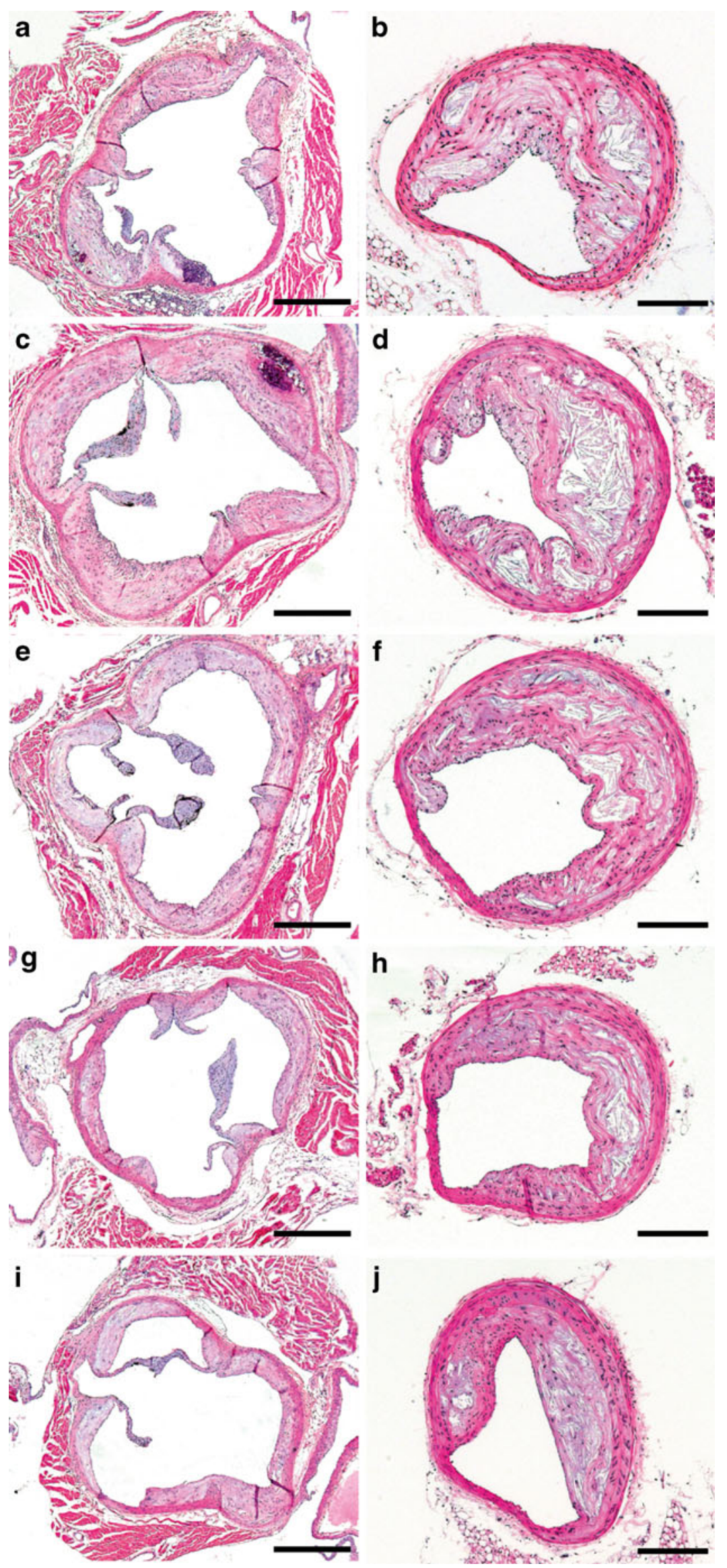
advanced atherosclerotic lesions in both the aortic root and BCA, as shown for the baseline group in Fig. 3a, b. At the aortic root, intimal area in the control progression group was 1.5 -fold $(p<0.001)$ higher compared with baseline mice (Fig. 2a). AdLDLr and AdA-I/AdLDLr gene transfer reduced aortic root intimal area by $28 \%(p<0.001)$ and $32 \%$ $(p<0.001)$, respectively, compared with the baseline group (Fig. 2a). No significant difference in aortic root intimal area was observed between AdLDLr and AdA-I/ AdLDLr treated mice. After AdA-I transfer, intimal area at the aortic root increased 1.3-fold $(p<0.01)$ compared with baseline mice and was not significantly lower than in control progression mice. At the level of the BCA, intimal area increased 1.6-fold $(p<0.001)$ during the 12 weeks after Adnull transfer or saline injection compared with baseline mice (Fig. 2b). After AdLDLr and AdA-I/AdLDLr gene transfer, intimal area at the BCA decreased by $25 \%(p<0.05)$ and by $17 \%$ $(p=\mathrm{NS})$, respectively, compared with baseline mice (Fig. 2b). Intimal area at the BCA in the AdA-I transfer group was not significantly different compared with the baseline group.

Regression of atherosclerosis following AdLDLr and AdA-I/AdLDLr gene transfer is not associated with negative remodeling Please see "Results" in the Electronic supplementary materials.

AdLDLr and AdA-I/AdLDLr gene transfer promote stability of complex atherosclerotic plaques in $\mathrm{LDLr}^{-/-}$mice.

To evaluate whether gene transfer also affects the composition and thereby the stability of advanced atherosclerotic plaques in the aortic root and BCA, the amount of smooth muscle cells (SMC), collagen, macrophages, $\mathrm{T}$ cells, and lipids the size of the necrotic core was quantified. Both relative and absolute data are presented in Table 2. Representative sections of the aortic root and BCA stained for different histological parameters are shown for all groups of mice in Figs. 4 and 5, respectively. At both locations, atherosclerotic lesions in baseline mice were complex and characterized by lipid-rich necrotic cores filled with cellular debris and covered by a fibrous cap consisting of smooth muscle cells, collagen, and a foam cell infiltrate (Figs. 4 and 5). At the aortic root, relative intimal SMC content was not significantly changed in the control progression and AdA-I groups compared with baseline mice, whereas AdLDLr and AdA-I/AdLDLr transfer increased the percentage SMC covered intimal area by 2.0 -fold $(p<0.01)$ (Table 2). At the level of the BCA, relative SMC-positive intimal area was not significantly different in the control progression and intervention groups compared with baseline (Table 2). Relative collagen area in the intima at the aortic root was similar in the control progression group compared with baseline mice, but was 1.8 -fold $(p<0.01), 1.5$-fold ( $p=\mathrm{NS})$, and 2.0-fold $(p<0.001)$ increased during the 12 weeks after AdA-I, AdLDLr, and AdA-I/AdLDLr transfer, respectively (Table 2). Similar results were obtained at the level of the BCA, where AdA-I, AdLDLr, and AdA-I/AdLDLr transfer increased the percentage of collagen covered intimal area by 1.5 -fold $(p=\mathrm{NS}), 1.7$-fold $(p<0.05)$, and 1.8 -fold $(p<0.05)$, respectively, compared with baseline mice (Table 2). Relative macrophage covered intimal area at the aortic root decreased 2.2-fold $(p<0.001), 2.9$-fold $(p<0.001), 4.8$-fold $(p<0.001)$, and 4.1 -fold $(p<0.001)$ during the 12 weeks following Adnull or saline injection, or transfer with AdA-I, AdLDLr, and AdA-I/AdLDLr, respectively, compared with baseline mice (Table 2). At the level of the BCA, relative macrophage-positive intimal area was not significantly affected in the control progression and AdA-I groups, but was 1.5-fold $(p=\mathrm{NS})$ and 1.4-fold $(p=\mathrm{NS})$ decreased after AdLDLr and AdA-I/AdLDLr transfer, respectively, compared with the baseline group. Intimal $T$ cell density, expressed as the number of $\mathrm{CD} 3$-positive cells per surface unit of intimal area, increased 2.1-fold $(p<0.01)$ and 1.7-fold $(p=\mathrm{NS})$ at the level of the aortic root in the control progression and AdA-I treated group, respectively, compared with baseline mice, but did not markedly change in the AdLDLr and AdA-I/AdLDLr groups. At the level of the $\mathrm{BCA}$, intimal $\mathrm{T}$ cell density increased during the 12 weeks after intervention, without any significant difference between all five groups (Table 2). Since lymphocyte infiltration predominantly occurs in the lamina adventitia, we also quantified $\mathrm{T}$ cell number in the adventitia. A decrease of adventitial $\mathrm{T}$ cell density was observed after AdLDLr and AdA-I/AdLDLr gene transfer. Please see "Results" in the Electronic supplementary materials for detailed analysis of these data. Relative lipid-positive intimal area at the aortic root did not differ between the five groups, but when expressed in absolute values, intimal lipid area was significantly increased in the control progression and AdAI groups, whereas AdLDLr and AdA-I/AdLDLr gene transfer resulted in a slight decrease of the absolute intimal lipid content, compared with baseline mice (Table 2). At the BCA, relative lipid-positive intimal area decreased 1.5-fold $(p=\mathrm{NS})$ and 1.4-fold $(p=\mathrm{NS})$ after AdLDLr and AdA-I/ AdLDLr gene transfer, which corresponds with a 1.8 -fold $(p<0.01)$ and 1.7 -fold $(p<0.05)$ lower relative area compared with control progression mice, respectively.

Quantification of necrotic core size, which is a determinant of plaque vulnerability, revealed that lesional necrotic core area was drastically reduced following AdLDLr and AdA-I/ AdLDLr gene transfer. Please see "Results" Electronic supplementary materials for extensive discussion of these data.

Quantification of intimal apoptosis by immunohistochemical staining for active caspase 3. Please see "Results" Electronic supplementary materials. 


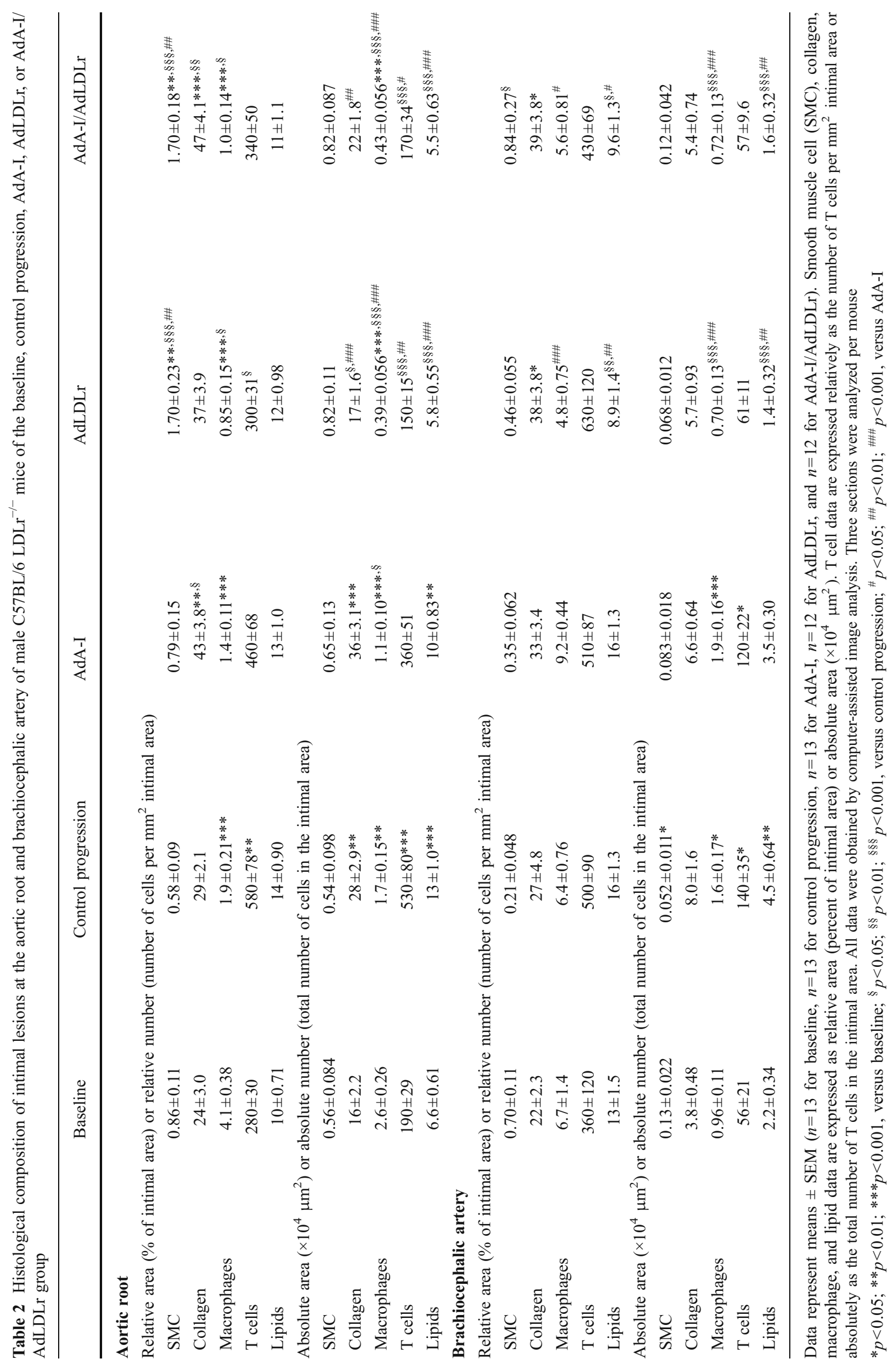




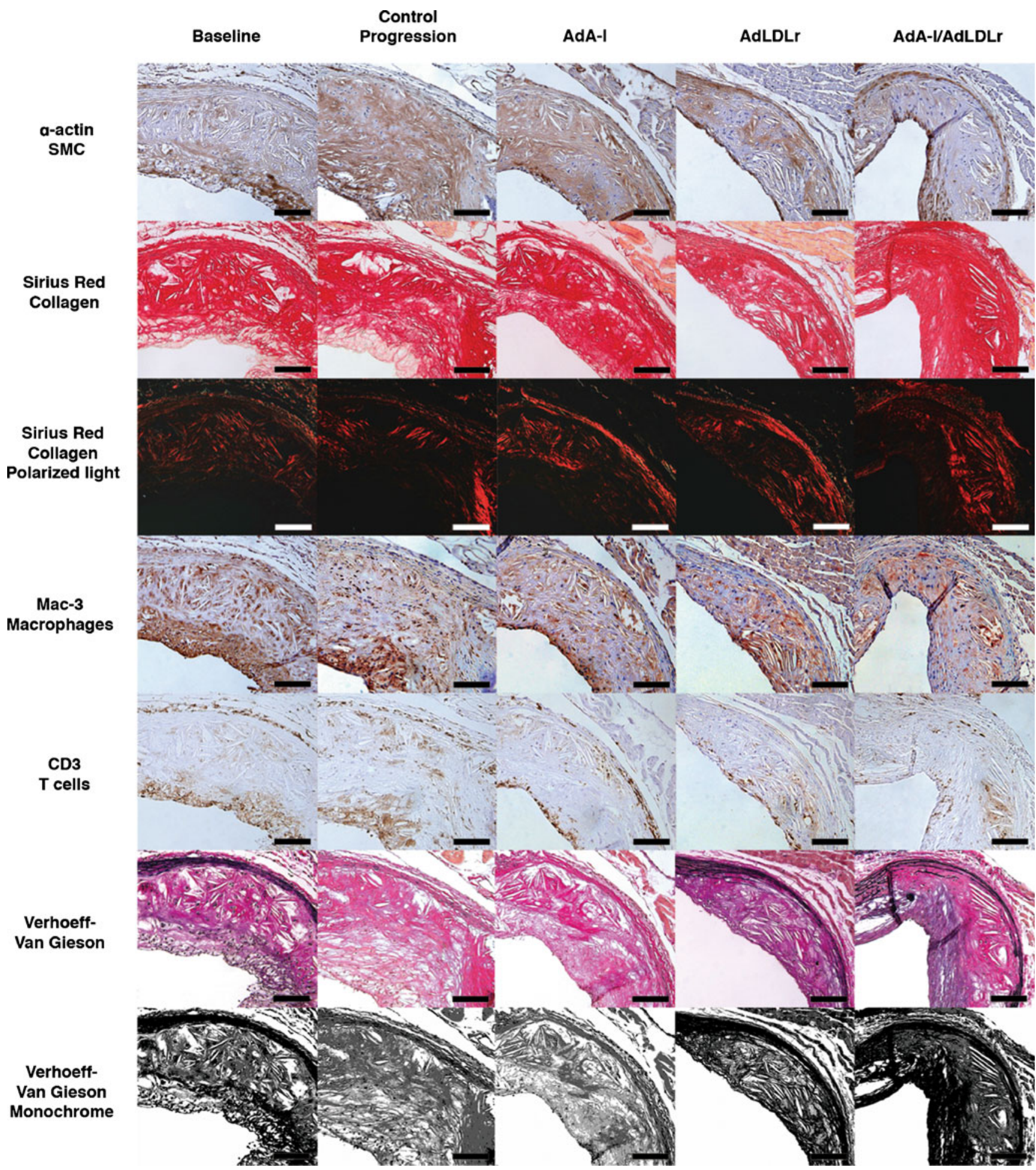

Fig. 4 Histological composition of intimal lesions located at the aortic root of male C57BL/6 $\mathrm{LDLr}^{-/-}$mice of the baseline group, the control progression group, and the AdA-I, AdLDLr, and AdA-I/ AdLDLr intervention groups. Representative photomicrographs show $\alpha$-actin-positive smooth muscle cells, Sirius red-stained collagen,
Sirius red-stained collagen viewed under polarized light, Mac-3positive macrophages, CD3-positive T cells, Verhoeff-Van Gieson staining, and Verhoeff-Van Gieson-stained images digitally processed into monochrome, which were used for quantification of intimal lipid content. Scale bar represents $100 \mu \mathrm{m}$ 
Gene expression profiling reveals changed expression of genes involved in lipid metabolism and reduced expression of inflammatory genes after AdLDLr or AdA-I/AdLDLr transfer

To further explore the effects of lipoprotein modifying interventions on the molecular composition of atherosclerotic plaques, the expression of atherosclerosis-related genes in the aortic root and BCA of baseline mice, control progression mice, and mice treated with AdA-I, AdLDLr, and AdA-I/ AdLDLr were analyzed using Mouse Atherosclerosis $\mathrm{RT}^{2}$ Profiler PCR Arrays (Electronic supplementary materials (Table 1)). This analysis shows that the expression of several genes involved in lipid transport and metabolism was significantly affected by AdLDLr or AdA-I/AdLDLr gene transfer. Both in the aortic root and BCA, ATP-binding cassette transporter A1 (Abca1), macrophage scavenger receptor 1 (Msr1), and apolipoprotein E (Apoe) were downregulated following AdLDLr and AdA-I/AdLDLr transfer compared with baseline mice. Several inflammatory genes were down-regulated in AdLDLr and AdA-I/AdLDLr treated mice including the chemokines MCP-1 (Cc12) and Gro-1 (Cxcl1), the chemokine receptors CD191 (Ccr1) and CD192 (Ccr2), and the cytokines interleukin-1 $\alpha$ (Il1a), interleukin$1 \beta$ (Illb), and interferon- $\gamma$ (Ifng). Down-regulation of inflammatory genes was mostly more pronounced in the combined AdA-I/AdLDLr gene transfer group compared with the AdLDLr group, as was the case for Ccl2, Cxcl1, Ccr1, and Il-1a (Electronic supplementary materials (Table 1)). In addition to inflammatory genes, the adhesion molecules intercellular adhesion molecule (Icam1), vascular cell adhesion molecule 1 (Vcam1), integrin $\alpha \mathrm{X}$ (Itgax), integrin $\beta 2$ (Itgb2), and p-selectin (Selp) were down-regulated after AdLDLr and AdA-I/AdLDLr transfer. Secreted phosphoprotein 1 (Spp1; also called osteopontin), which has an important role in plaque calcification, was also significantly downregulated at the aortic root and BCA after AdLDLr and AdAI/AdLDLr gene transfer (Electronic supplementary materials (Table 1)). AdA-I gene transfer alone did not have any major impact on gene expression in lesions.

LDL lowering gene transfer may be accompanied by a shift of macrophages toward a more favorable M2 phenotype. Please see "Results" in the Electronic supplementary materials.

Regression of atherosclerosis following AdLDLr and AdA-I/AdLDLr gene transfer is not accompanied by an upregulation of the chemokine receptor CCR7 in atherosclerotic lesions. Please see "Results" in the Electronic supplementary materials.

\section{Discussion}

The main findings of the present study are that (1) a drastic reduction of non-HDL cholesterol levels following gene transfer with LDLr expressing E1E3E4-deleted adenoviral vectors in $\mathrm{C} 57 \mathrm{BL} / 6 \mathrm{LDLr}^{-/-}$mice causes pronounced regression of advanced atherosclerotic lesions in the aortic root and brachiocephalic artery; (2) AdLDLr gene transfer promotes the stability of advanced atherosclerotic lesions as evidenced by an increase in collagen content, a decrease in lesion macrophage content, a decrease in necrotic core area, and a reduced expression of inflammatory genes; (3) increased HDL cholesterol following apo A-I gene transfer elevates collagen content in lesions, but does not induce regression; and (4) HDL raising gene transfer on top of AdLDLr transfer results in additive effects at the level of gene expression, particularly on inflammatory gene expression.

Promoting regression and stabilization of advanced atherosclerotic lesions is a major goal for decreasing morbidity and mortality from ischemic cardiovascular diseases [2]. In the current study, we studied lesions at two different topographic sites, namely in the aortic root, an elastic artery where lesions develop earliest in mice, and in the brachiocephalic artery [13], a muscular artery prone to rapid development of complex lesions. Feeding an atherogenic diet to male C57BL/6 $\mathrm{LDLr}^{-/-}$mice for a period of 6 months resulted at these two sites in the development of advanced lesions in the baseline group containing lipid-rich necrotic cores, cholesterol clefts, fibrous caps, and a foam cell infiltrate. In this study, we investigated the relative potential of LDL lowering and HDL raising interventions to induce regression and plaque stabilization. Gene transfer with AdLDLr, an E1E3E4-deleted adenoviral vector containing a potent hepatocyte-specific expression cassette [18], constitutes, to the best of our knowledge, the most potent lipid lowering intervention. Non-HDL cholesterol levels were reduced by more than $95 \%$. A unique feature of the current study was that regression of advanced atherosclerotic lesions was investigated in an experimental protocol with maintenance of the atherogenic diet throughout the experiment. This is in sharp contrast with other regression studies in mice, rabbits, swine, and non-human primates in which lipid lowering was achieved via withdrawal of the dietary stimulus or in which lipoprotein modifying interventions were accompanied by dietary manipulations [4, 13-15]. Importantly, modification of the diet may affect atherosclerotic lesions independent of cholesterol lowering, rendering the interpretation of such studies complex. For example, withdrawal of saturated fat may have beneficial effects on atherosclerosis independent of lipoprotein cholesterol levels by decreasing selective cholesterol uptake in the arterial wall [21] and by reducing vascular inflammation [22]. Since AdLDLr gene transfer is a highly selective lipid lowering intervention, lipid lowering-independent effects did not contribute to regression in the current study. Atheroma volume was decreased 


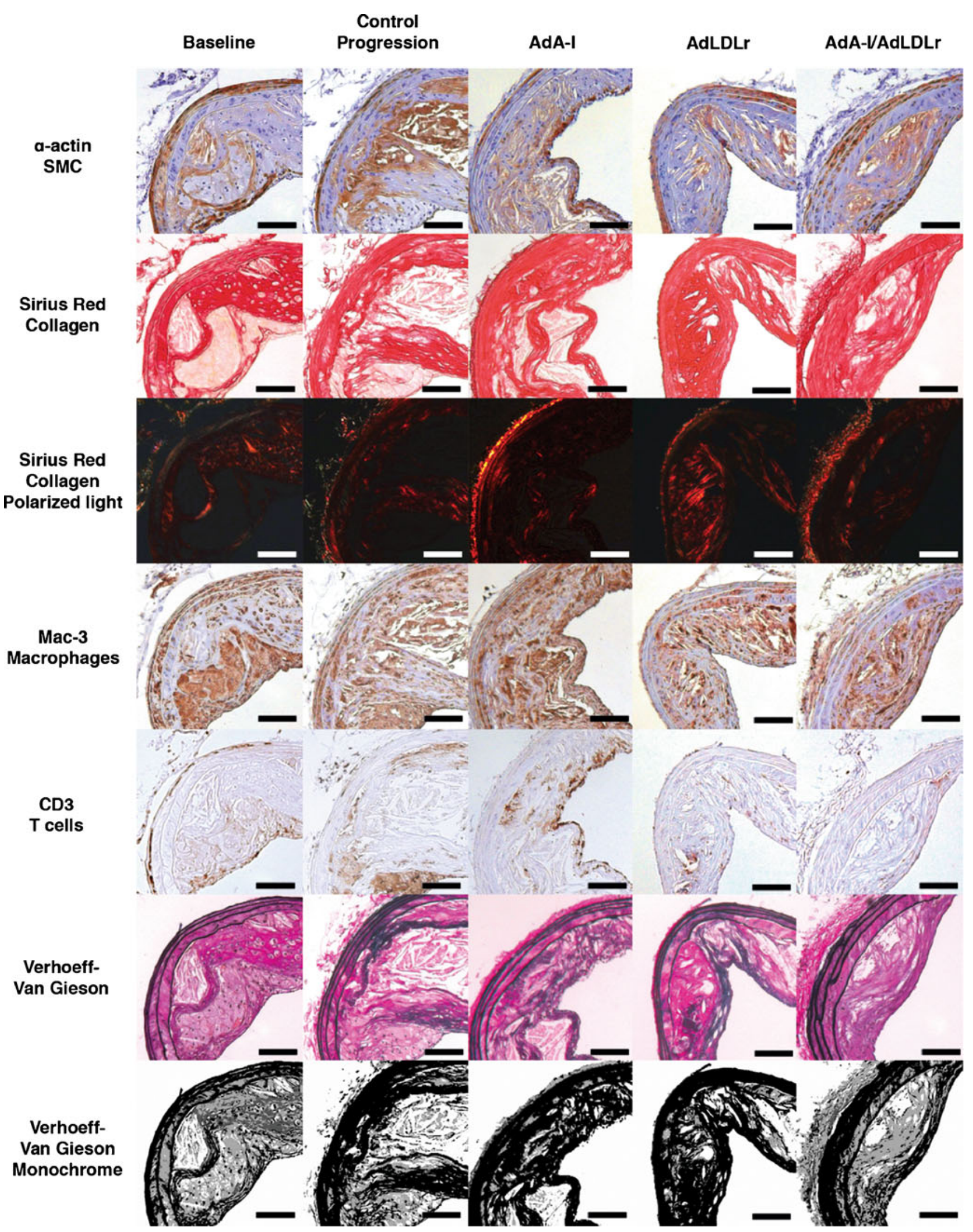


4Fig. 5 Histological composition of intimal lesions located at the brachiocephalic artery of male $\mathrm{C} 57 \mathrm{BL} / 6 \mathrm{LDLr}^{-/-}$mice of the baseline group, the control progression group, and the AdA-I, AdLDLr, and AdA-I/AdLDLr intervention groups. Representative photomicrographs show $\alpha$-actin-positive smooth muscle cells, Sirius red-stained collagen, Sirius red-stained collagen viewed under polarized light, Mac-3-positive macrophages, CD3-positive T cells, Verhoeff-Van Gieson staining, and Verhoeff-Van Gieson-stained images digitally processed into monochrome, which were used for quantification of intimal lipid content. Scale bar represents $100 \mu \mathrm{m}$

by $28 \%$ and $25 \%$ in the aortic root and in the brachiocephalic artery, respectively, following AdLDLr gene transfer. To put these numbers into perspective, a comparison with atherosclerosis regression in humans is interesting. The median reduction of plaque volume in the ASTEROID trial was $6.8 \%$ following a $53 \%$ reduction of LDL cholesterol levels to $61 \mathrm{mg} / \mathrm{dl}$ induced by high-dose rosuvastatin therapy [3].

Besides the pronounced reduction of atheroma volume, AdLDLr and AdA-I/AdLDLr gene transfer were accompanied by important histological and molecular changes known to be associated with increased plaque stability [23]. Similarly as has been reported in rabbit lesions after dietary lipid lowering [23], intimal collagen content was significantly increased in both lipid lowering groups. Correspondingly, expression of interferon- $\gamma$, a $\mathrm{T}$ cell lymphokine known to reduce collagen biosynthesis by smooth muscle cells [24], was decreased. AdLDLr and AdA-I/AdLDLr gene transfer decreased lesional macrophage content, which was associated with a decreased intimal expression of Abca1, Apoe, and Msr1, all known to be expressed by macrophages [25-27]. Furthermore, apo B lowering gene transfer decreased intimal expression of cytokines, chemokines, chemokine receptors, and adhesion molecules. This indicates that inflammation in advanced atherosclerotic lesions can be reduced by lipid lowering itself. Other plaque stabilizing effects of apo B lowering gene transfer include a reduction of intimal expression of matrix metalloproteinase-3 (MMP-3 or stromelysin-1) and secreted phosphoprotein 1 (Spp1 or osteopontin), which is involved in weakening the connective tissue matrix [28] and in regulating plaque calcification [29], respectively.

Elevated HDL cholesterol following AdA-I gene transfer increased intimal collagen content compared with baseline mice but did not induce regression of atherosclerosis. Lesional macrophage content was reduced following AdA-I gene transfer in the aortic root but not in the brachiocephalic artery. Continued progression of advanced atherosclerotic lesions notwithstanding increased HDL cholesterol has also been observed in a another murine model by Rong et al. [30]. In a recent study on the effect of HDL raising gene transfer on advanced atherosclerotic lesions in rabbits, we showed that increased HDL choles- terol following gene transfer resulted in an inhibition of progression of atherosclerosis and in a reduction of neutral lipid content in the intima compared with baseline [31]. The absence of similar effects of AdA-I gene transfer on advanced lesions in the current study may be related to the very high levels of pro-atherogenic lipoproteins (nonHDL cholesterol levels of approximately $650 \mathrm{mg} / \mathrm{dl}$ ), which override the effects of increased HDL cholesterol. Furthermore, baseline HDL cholesterol levels in C57BL/6 $\mathrm{LDLr}^{-/}$ mice fed an atherogenic diet were significantly higher than in $\mathrm{C} 57 \mathrm{BL} / 6$ apo $\mathrm{E}^{-/-}$mice [32] or in wild-type $\mathrm{C} 57 \mathrm{BL} / 6$ mice [33] and consequently, the relative increase of HDL cholesterol following apo A-I gene transfer was less pronounced in the current study than observed in other models $[32,33]$.

Additive effects of raising HDL cholesterol levels in the setting of a drastic reduction of apo B containing lipoproteins were observed at the molecular level. The reduction of intimal expression of several inflammatory genes was significantly more pronounced in the AdA-I/ AdLDLr group compared with the AdLDLr group, which is consistent with the anti-inflammatory effects of HDL. No major differences in cellular composition of atherosclerotic lesions were observed between the AdLDLr group and the AdA-I/AdLDLr group, particularly in the aortic root. Therefore, comparison of gene expression profiling results between these two groups highlights differences at the cellular level and not simply differences of cellularity of the plaque. The less pronounced increase of HDL cholesterol in the AdA-I/AdLDLr group compared with the AdA-I group may be partially explained by catabolism of apo $\mathrm{E}$ containing HDL particles via the LDL receptor in the AdA-I/AdLDLr group but is likely predominantly due to a reduced transfer of free cholesterol from LDL to HDL in the setting of low LDL cholesterol levels.

The current study represents a head to head comparison of LDL lowering and HDL raising gene transfer strategies using vectors with exactly the same transcriptional regulatory sequences. Nevertheless, the relative effect of human apo A-I transfer on HDL cholesterol levels was moderate compared with the drastic lowering of pro-atherogenic lipoproteins after AdLDLr gene transfer. A further limitation of the current study is that an increase of triglycerides occurred after AdA-I transfer. In contrast, no change of triglycerides was previously observed after human apo A-I transfer in C57BL/6 apo $\mathrm{E}^{-/-}$mice fed normal chow [34]. Therefore, the increase of triglycerides after human apo A-I transfer is model and diet dependent. Enrichment of lipoproteins with triglycerides could have hampered lesion regression.

In conclusion, drastic lowering of apo B containing lipoproteins following LDLr gene transfer in hypercholesterolemic mice results in a pronounced regression and a 
more stable phenotype of advanced atherosclerotic lesions. HDL raising gene transfer on top of AdLDLr transfer results in additive effects at the level of gene expression, particularly on inflammatory gene expression.

Acknowledgments This work was supported by grants G.0533.08 and G.0599.09N of the Fonds voor Wetenschappelijk OnderzoekVlaanderen. The Center for Molecular and Vascular Biology is supported by the Excellentiefinanciering KU Leuven (EF/05/013). Eline Van Craeyveld is a Research Assistant of the Fonds voor Wetenschappelijk Onderzoek-Vlaanderen. Stephanie C. Gordts is a Research Assistant of the Agentschap voor Innovatie door Wetenschap en Technologie (IWT). Frank Jacobs is a postdoctoral fellow of the Fonds voor Wetenschappelijk Onderzoek-Vlaanderen.

\section{Disclosures/Conflicts of interest None.}

Open Access This article is distributed under the terms of the Creative Commons Attribution Noncommercial License which permits any noncommercial use, distribution, and reproduction in any medium, provided the original author(s) and source are credited.

\section{References}

1. Aikawa M, Libby P (2004) Lipid lowering therapy in atherosclerosis. Semin Vasc Med 4:357-366

2. Williams KJ, Feig JE, Fisher EA (2008) Rapid regression of atherosclerosis: insights from the clinical and experimental literature. Nat Clin Pract Cardiovasc Med 5:91-102

3. Nissen SE, Nicholls SJ, Sipahi I, Libby P, Raichlen JS, Ballantyne CM, Davignon J, Erbel R, Fruchart JC, Tardif JC et al (2006) Effect of very high-intensity statin therapy on regression of coronary atherosclerosis: the ASTEROID trial. JAMA 295:15561565

4. Corti R, Osende JI, Fallon JT, Fuster V, Mizsei G, Jneid H, Wright SD, Chaplin WF, Badimon JJ (2004) The selective peroxisomal proliferator-activated receptor-gamma agonist has an additive effect on plaque regression in combination with simvastatin in experimental atherosclerosis: in vivo study by high-resolution magnetic resonance imaging. J Am Coll Cardiol 43:464-473

5. Crisby M, Nordin-Fredriksson G, Shah PK, Yano J, Zhu J, Nilsson J (2001) Pravastatin treatment increases collagen content and decreases lipid content, inflammation, metalloproteinases, and cell death in human carotid plaques: implications for plaque stabilization. Circulation 103:926-933

6. Gordon DJ, Rifkind BM (1989) High-density lipoprotein - the clinical implications of recent studies. N Engl J Med 321:13111316

7. Nicholls SJ, Tuzcu EM, Sipahi I, Grasso AW, Schoenhagen P, Hu T, Wolski K, Crowe T, Desai MY, Hazen SL et al (2007) Statins, high-density lipoprotein cholesterol, and regression of coronary atherosclerosis. JAMA 297:499-508

8. Taylor AJ, Sullenberger LE, Lee HJ, Lee JK, Grace KA (2004) Arterial Biology for the Investigation of the Treatment Effects of Reducing Cholesterol (ARBITER) 2: a double-blind, placebocontrolled study of extended-release niacin on atherosclerosis progression in secondary prevention patients treated with statins. Circulation 110:3512-3517

9. Nissen SE, Tsunoda T, Tuzcu EM, Schoenhagen P, Cooper CJ, Yasin M, Eaton GM, Lauer MA, Sheldon WS, Grines CL et al (2003) Effect of recombinant ApoA-I Milano on coronary atherosclerosis in patients with acute coronary syndromes: a randomized controlled trial. Jama 290:2292-2300

10. Tardif JC, Gregoire J, L'Allier PL, Ibrahim R, Lesperance J, Heinonen TM, Kouz S, Berry C, Basser R, Lavoie MA et al (2007) Effects of reconstituted high-density lipoprotein infusions on coronary atherosclerosis: a randomized controlled trial. Jama 297:1675-1682

11. Badimon JJ, Badimon L, Fuster V (1990) Regression of atherosclerotic lesions by high density lipoprotein plasma fraction in the cholesterol-fed rabbit. J Clin Invest 85:1234-1241

12. Tangirala RK, Tsukamoto K, Chun SH, Usher D, Pure E, Rader DJ (1999) Regression of atherosclerosis induced by liver-directed gene transfer of apolipoprotein A-I in mice. Circulation 100:1816-1822

13. MacDougall ED, Kramer F, Polinsky P, Barnhart S, Askari B, Johansson F, Varon R, Rosenfeld ME, Oka K, Chan L et al (2006) Aggressive very low-density lipoprotein (VLDL) and LDL lowering by gene transfer of the VLDL receptor combined with a low-fat diet regimen induces regression and reduces macrophage content in advanced atherosclerotic lesions in LDL receptordeficient mice. Am J Pathol 168:2064-2073

14. Strong JP, Bhattacharyya AK, Eggen DA, Stary HC, Malcom GT, Newman WP 3rd, Restrepo C (1994) Long-term induction and regression of diet-induced atherosclerotic lesions in rhesus monkeys. II. Morphometric evaluation of lesions by light microscopy in coronary and carotid arteries. Arterioscler Thromb 14:2007-2016

15. Reis ED, Li J, Fayad ZA, Rong JX, Hansoty D, Aguinaldo JG, Fallon JT, Fisher EA (2001) Dramatic remodeling of advanced atherosclerotic plaques of the apolipoprotein E-deficient mouse in a novel transplantation model. J Vasc Surg 34:541-547

16. De Geest B, Van Linthout S, Lox M, Collen D, Holvoet P (2000) Sustained expression of human apolipoprotein A-I after adenoviral gene transfer in C57BL/6 mice: role of apolipoprotein A-I promoter, apolipoprotein A-I introns, and human apolipoprotein E enhancer. Hum Gene Ther 11:101-112

17. Van Linthout S, Collen D, De Geest B (2002) Effect of promoters and enhancers on expression, transgene DNA persistence, and hepatotoxicity after adenoviral gene transfer of human apolipoprotein A-I. Hum Gene Ther 13:829-840

18. Jacobs F, Snoeys J, Feng Y, Van Craeyveld E, Lievens J, Armentano D, Cheng SH, De Geest B (2008) Direct comparison of hepatocyte-specific expression cassettes following adenoviral and nonviral hydrodynamic gene transfer. Gene Ther 15:594-603

19. Jacobs F, Van Craeyveld E, Feng Y, Snoeys J, De Geest B (2008) Adenoviral low density lipoprotein receptor attenuates progression of atherosclerosis and decreases tissue cholesterol levels in a murine model of familial hypercholesterolemia. Atherosclerosis 201:289-297

20. Van Linthout S, Lusky M, Collen D, De Geest B (2002) Persistent hepatic expression of human apo A-I after transfer with a helpervirus independent adenoviral vector. Gene Ther 9:1520-1528

21. Seo T, Qi K, Chang C, Liu Y, Worgall TS, Ramakrishnan R, Deckelbaum RJ (2005) Saturated fat-rich diet enhances selective uptake of LDL cholesteryl esters in the arterial wall. J Clin Invest 115:2214-2222

22. Kim F, Pham M, Luttrell I, Bannerman DD, Tupper J, Thaler J, Hawn TR, Raines EW, Schwartz MW (2007) Toll-like receptor-4 mediates vascular inflammation and insulin resistance in diet-induced obesity. Circ Res 100:1589-1596

23. Aikawa M, Rabkin E, Okada Y, Voglic SJ, Clinton SK, Brinckerhoff CE, Sukhova GK, Libby P (1998) Lipid lowering by diet reduces matrix metalloproteinase activity and increases collagen content of rabbit atheroma: a potential mechanism of lesion stabilization. Circulation 97:2433-2444 
24. Amento EP, Ehsani N, Palmer H, Libby P (1991) Cytokines and growth factors positively and negatively regulate interstitial collagen gene expression in human vascular smooth muscle cells. Arterioscler Thromb 11:1223-1230

25. Feng B, Tabas I (2002) ABCA1-mediated cholesterol efflux is defective in free cholesterol-loaded macrophages. Mechanism involves enhanced ABCA1 degradation in a process requiring full NPC1 activity. J Biol Chem 277:43271-43280

26. O'Brien KD, Deeb SS, Ferguson M, McDonald TO, Allen MD, Alpers CE, Chait A (1994) Apolipoprotein E localization in human coronary atherosclerotic plaques by in situ hybridization and immunohistochemistry and comparison with lipoprotein lipase. Am J Pathol 144:538-548

27. Manning-Tobin JJ, Moore KJ, Seimon TA, Bell SA, Sharuk M, Alvarez-Leite JI, de Winther MP, Tabas I, Freeman MW (2009) Loss of SR-A and CD36 activity reduces atherosclerotic lesion complexity without abrogating foam cell formation in hyperlipidemic mice. Arterioscler Thromb Vasc Biol 29:19-26

28. Henney AM, Wakeley PR, Davies MJ, Foster K, Hembry R, Murphy G, Humphries S (1991) Localization of stromelysin gene expression in atherosclerotic plaques by in situ hybridization. Proc Natl Acad Sci USA 88:8154-8158

29. Dhore CR, Cleutjens JP, Lutgens E, Cleutjens KB, Geusens PP, Kitslaar PJ, Tordoir JH, Spronk HM, Vermeer C, Daemen MJ (2001) Differential expression of bone matrix regulatory proteins in human atherosclerotic plaques. Arterioscler Thromb Vasc Biol 21:1998-2003
30. Rong JX, Li J, Reis ED, Choudhury RP, Dansky HM, Elmalem VI, Fallon JT, Breslow JL, Fisher EA (2001) Elevating highdensity lipoprotein cholesterol in apolipoprotein E-deficient mice remodels advanced atherosclerotic lesions by decreasing macrophage and increasing smooth muscle cell content. Circulation 104:2447-2452

31. Van Craeyveld E, Lievens J, Jacobs F, Feng Y, Snoeys J, De Geest B (2009) Apolipoprotein A-I and lecithin:cholesterol acyltransferase transfer induce cholesterol unloading in complex atherosclerotic lesions. Gene Ther 16:757-765

32. Feng Y, Van Craeyveld E, Jacobs F, Lievens J, Snoeys J, De Geest B (2009) Wild-type apo A-I and apo A-I(Milano) gene transfer reduce native and transplant arteriosclerosis to a similar extent. $\mathrm{J}$ Mol Med 87:287-297

33. Van Linthout S SF, Graiani G, Miteva K, Peng J, Van Craeyveld E, Meloni M, Tölle M, Escher F, Subasigüller A, Doehner, Quaini F, De Geest B, Schultheiss HP, Tschöpe C (2010) Downregulation of endothelial TLR4 signalling after apo A-I gene transfer contributes to improved survival in an experimental model of lipopolysaccharide-induced inflammation. J Mol Med (in press)

34. Feng Y, Lievens J, Jacobs F, Hoekstra M, Van Craeyveld E, Gordts SC, Snoeys J, De Geest B (2010) Hepatocyte-specific ABCA1 transfer increases HDL cholesterol but impairs HDL function and accelerates atherosclerosis. Cardiovasc Res 88:376385 